\title{
Article
}

\section{Surround Modulation properties of tectal neurons in pigeon characterized by moving and flashed stimuli}

\author{
Xiaoke Niu' ${ }^{1,2}$, Shuman Huang ${ }^{1}$, Minjie Zhu ${ }^{1}$, Zhizhong Wang ${ }^{1}$ and Li Shi ${ }^{1,3, *}$ \\ 1 Henan Key Laboratory of Brain Science and Brain-Computer Interface Technology, School of \\ Electrical Engineering, Zhengzhou University, Zhengzhou, 450001, China 1; e-mail@e-mail.com \\ 2 College of Basic Medicine, Zhengzhou University, Zhengzhou, 450001, China \\ 3 Department of Automation, Tsinghua University, Beijing, 100000, China \\ * Correspondence: shilizzu@163.com
}

\begin{abstract}
Simple Summary: Surround modulation is a basic visual attribute of sensory neurons in many species and sensory modalities, which have been extensively characterized in mammal primary visual cortex, LGN and superior colliculus, yet too little attention has been paid to birds, which have a highly developed visual system. We made a systematic analysis on surround modulation properties of tectal neurons in pigeons. This study complements existing surrounding modulation properties in non-mammalian species, and deepens the understanding of mechanisms of figure-background segmentation performed by animals.
\end{abstract}

\begin{abstract}
Surround modulation is a phenomenon whereby costimulation of the extra-classical receptive field and classical receptive field would modulate the visual responses induced individually by classical receptive field. However, there lacks systematic study about surround modulation properties existing in avian optic tectum. In this study, neuronal activities are recorded from pigeon optic tectum, and the responses to moving and flashed squares and bars of different sizes are compared. The statistical results showed that most tectal neurons presented surround suppression as stimuli size grew larger both in moving and flashed paradigms, and the suppression degree induced by larger flashed square was comparable with that by moving one when it crossed near the cell's RF center, which corresponds to fully surrounding condition. The suppression degree grew weaker when the stimuli move across the RF border, which corresponds to partially surrounding condition. Meanwhile, the fully surround suppression induced by flashed square was also more intense than partially surrounded by flashed bars. The results provide new insight for understanding the spatial arrangement of lateral inhibitions from feedback or feedforward streams, which would help to make clear the generation mechanism of surround modulation found in avian optic tectum.
\end{abstract}

Keywords: surround suppression, extra-classical receptive field, optic tectum, size tuning

\section{Introduction}

The visual response to classical receptive field (CRF) stimuli can be modulated by their surrounding field, where stimulating alone cannot elicit spikes [1-3]. This phenomenon is called "surround modulation", which was generally suppressive [4-13] rather than facilitative [14-18], and is related to visual feature integration and figure-ground segregation[9,19]. The fundamental nature of surround modulation has been fairly described in mammal primary visual cortex (V1), LGN and superior colliculus (SC), whereas the latter of which was homologous to optic tectum (OT) in avian. Relatively fewer studies have been devoted to non-mammalian species, e.g. birds, who have evolved highly advanced visual systems [20,21], and the pigeon has been widely used as an animal model in the majority of visual neuroscience studies [22]. As far as we know, there lacks detailed quantitative description of surrounding modulation properties of tectal neurons in pigeons.

The existing studies carried in mammals have concluded that the surround modulation in V1 and LGN was selective to visual features, such as orientation, spatial fre- 
quency $[23,24]$. Those properties may relate to the nature of neurons in V1 and LGN, which was selective to orientation or spatial frequency. The response modulations in SC are much more noticeable to direction contrast compared to phase, temporal frequency, and static orientation contrast [25]. Subsequent research shows that the surround suppression could sharpen the orientation tuning [26] and enhance the single neuron's orientation selectivity $[27,28]$ in $V 1$, as well as enhanced connection strength and the network structural properties among local population networks in V1 [29]. The most recent work further revealed dynamic surround suppression presented in SC, which was affected by temporal context (adaptation) [30].

In avian, the neuron in OT was mostly preferred to respond to motion and a small number of neurons presented direction selective. Earlier study on surround modulation has reported that tectal response to motion was modulated by large moving background [31]. The suppression was strongest when the moving direction of the center was the same with that of the background [32,33]. Tectal neurons also responded to spatial contrasting stimuli, when the contrast between center and background was the direction of motion rather than the orientation, and when the center was looming and the background receding but not when the center was receding and the background looming [34]. The surround suppression of tectal response was less when the surrounding elements all moved in one direction (homogeneously moving), compared with nonhomogeneously moving elements [35]. A recent work further reported that response to single static flashed bar of tectal neuron could also be inhibited by surrounding flashed bars, and the suppression was strong when the luminance between the center and surrounding was the same, but less than when the motion direction between the center and surrounding was the same [36]. The above studies explored the surround modulation properties in different aspects. Nevertheless, it was still unclear whether surround modulation by moving stimuli presented the same with that by flashed ones in OT, and how differently they presented between the suppression induced by fully surrounding stimuli and by partially surrounding stimuli.

To make clear the above questions, we recorded multi-units from middle layer OT in anesthetized pigeons with multi-electrode array (MEA), and analyzed the tetcal response to different sizes of flashed squares and bars, as well as response to moving stimuli of the same levels of sizes. The results showed that most tectal response increased first and then inhibited as the size of the stimuli grew larger in both flashed and moving conditions. The modulation trend was much consistent with previous reports taken in mammals and birds. What's more, we found the following findings to be novel. The suppression degree induced by flashed square was not significantly different from that by moving square when it crossed near the cell's RF center. The suppression degree grew weaker when taking into account the response to moving stimuli crossing the RF border. What's more, the surround suppression induced by bar was weaker than that by squares. The above results implied that the fully surround suppression was larger than partially surrounded. 


\section{Materials and Methods}

\section{Animal preparation}

Our data were recorded from 17 pigeons (Columba livia, homing pigeon, either sex, weighing 300-500 g) maintained by the Animal Center of Zhengzhou University. The study was conducted according to guidelines of the National Institutes of Health and the Declaration of Helsinki. All experiments were approved by the Animal Care and Use Committee of Zhengzhou University (No SYXK 2019-0002).

The experimental preparation has been described previously (Niu et al., 2020). Each pigeon was anesthetized with urethane (1.8-2.2 g/ $\mathrm{kg}$ body weight) and restrained in a stereotaxic apparatus (model ST-5ND-B; Chengdu Instrument, Chengdu, China). A small dorso-lateral tectum region on the left side was exposed for craniotomy (Letelier, 2004). Neuronal activity was recorded with an MEA that consisted of 16 polyimide-insulated platinum/iridium microwires, the same type with previous study (Niu et al., 2020). The recording sites were verified by the following histological reconstruction.

The experimenter assessed their eye movements by intermittently monitoring the anesthetized pigeons during data recording, and no eye movement was observed (Niu et al., 2006). Furthermore, the classical receptive fields (CRF) of the recorded unit were obtained at intervals of $30 \mathrm{~min}$ by the experimenter, and no shift of CRF location was observed.

\section{Visual stimuli and electrophysiological recordings}

Visual stimuli were generated with the ViSaGe MKII visual stimulus generator (Cambridge Research Systems, Rochester, England) and presented on a gamma-calibrated Sony monitor (monitor size: $300 \times 400 \mathrm{~mm}$; resolution: $480 \times 640$ pixels; frame rate: $100 \mathrm{~Hz}$ ). The monitor was positioned $40 \mathrm{~cm}$ in front of the pigeon's right eye. The center of the RF was determined at each recording site by first mapping rough and detailed RF in the same way introduced in the previous study (Niu et al., 2020). After that, the following types of stimuli were used to test the surround modulation properties of tectal neurons.

1) Flashed stimuli. Flashed squares of different side lengths were used to map size tuning curve of each recorded tectal neuron. And the size of CRF (indicated with $R_{c}$ ) and eCRF (denoted by $\mathrm{Re}$ ) were determined under this stimuli condition. Flashed bars keep one side length fixed (set to $\mathrm{R}_{\mathrm{c}}$ or $\mathrm{R}_{\mathrm{e}}$ ), and the other side changes in the same levels as the side length of the previous squares. Each group of stimuli flashed at the center of the neuron's RF on a gray background in pseudo-randomized order for 20 repetitions. Each stimulus lasted for $100 \mathrm{~ms}$ with an interstimulus interval of $100 \mathrm{~ms}$.

2) Moving stimuli. The size of moving squares was the same with the above flashed stimuli. Note that the moving path was set long enough assuring the neuron don't response at the beginning and the end of each motion.

The signals were collected with a Cerebus system (Blackrock® Microsystems, Salt Lake City, UT, USA) and amplified 4000×. The spikes were detected by thresholding the band-pass filtered (Second-order Butterworth) raw signals between $250 \mathrm{~Hz}$ and $5 \mathrm{kHz}$ with a sampling rate of $30 \mathrm{kHz}$. The threshold value was set to 5.25 times the standard deviation of the band passed signal. Data analysis

Data analysis was performed in MATLAB (The MathWorks). The mean firing rate under each stimulus was first calculated. The size tuning curve based on mean firing rate was then fitted with the Piecewise Gaussian model [37] as follows, $R(x)=\left\{\begin{array}{c}K e^{-(x-a)^{2} / b^{2}}+d, x>=a \\ (K+d) e^{-(x-a)^{2} / c^{2}}, x<=a\end{array}\right.$

where $x$ is the stimulus size (side length of squares, length of the varying side of the bar), $a$ is the CRF size, $\mathrm{K}$ is amplitude of the Gaussian function, $b$ and $c$ are width, $d$ is offset of asymptotic response from spontaneous activity. Generally, the size tuning curve 
was always fitted with the difference-of-Gaussians (DoG) model [7,38,39]. However, the data in our study were not consistent with its assumptions. Thus the data were divided into two phases, ascending and descending limb, the fitting parameters $K, b$, and $d$ of the latter were first evaluated considering enough data points. The ascending limb of the size tuning curve (dotted line) was then fitted using $\mathrm{K}$, $\mathrm{d}$, and the free parameter $\mathrm{c}$. All values were optimized to minimize sum of squared error to access a good fit, which was assessed using mean Adjust-R2. Each fitted size tuning curve was normalized to the maximum value.

When fitting curves, fits were weighted with the reciprocal of the variance and were optimized to minimize sum of squared error.

The suppression index (SI) was finally calculated with $\mathrm{K} /(\mathrm{K}+\mathrm{d})$ to quantify the strength before the normalization. The index takes a value between 0 (indicates no suppression) and 1 (indicates complete suppression). The statistical analysis was carried out using Wilcoxon signed-rank test for data of same size and Wilcoxon rank sum test for data of different size. The statistical comparison graphs were drawn using Origin 2019b.

\section{Results}

We totally recorded 137 multi-units with obvious parafoveal RF from middle layer OT of 17 pigeons. Neurons from 31 recording sites those presented unstable response to repeat stimuli were discarded. The statistical results were based on the left 106 recording sites. For each part of the following results, we only presented the detailed results of an example from a single recording site.

Surround modulation properties by flash stimuli.

Surround modulation properties were first tested with flashed squares of different sizes, located at their RF centers (indicated with red dot in Fig. 1a), at 57 recording sites. Most cells (40/57=70.2\%) presented surround suppression property. That is, the response increased first and then inhibited as the stimuli size grew larger. The detail results of an example from a single recording site were summarized in Fig.1. The mean firing rate under each size of square (black squares shown in Fig.1a) was calculated and shown in Fig. 1b. The raw data to different sizes were then fitted with the Piecewise Gaussian model (Eq. 1) to obtain the size-tuning curve, as shown in Fig. 1c, in which the fitted parameters were all presented. According to the definitions of CRF (the size of the bar evoking the strongest neural response, denoted by $R_{c}$ ) and eCRF (the size of the smallest bar suppressing response, indicated with $R_{e}$ ), the size of CRF and eCRF were obtained from the curve. The distribution of the estimated $R_{c}$ and $R_{e}$ for all the selected 40 recording sites were shown in Fig.1d \& e. 


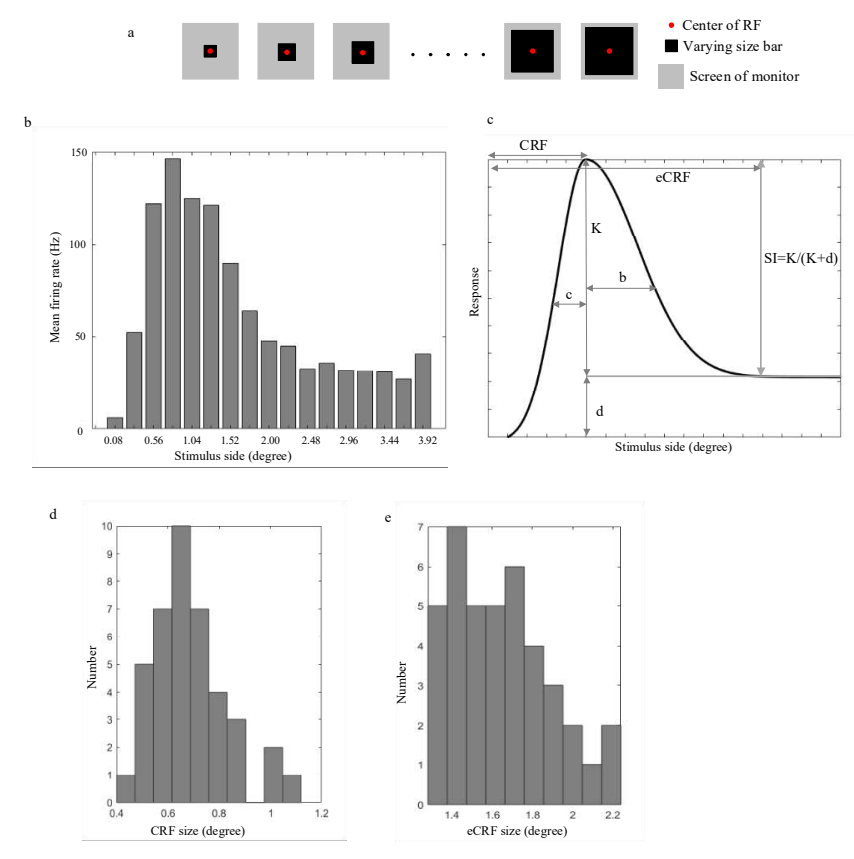

Figure 1. Size tuning curve of flashed squares stimuli as well as the estimated parameters. (a) Flashed squares (denoted by black square) of different sizes. The larger gray square indicates the scope of the screen. The middle red dot denotes the RF center; (b) The original mean firing rates under each stimulus shown in a; (c) The fitted size tuning curve (adjust-R2 $=0.9462$, left; adjust-R2 $=0.8628$, right) of data shown in B and explanation of each fitting parameter as well as the estimated CRF and eCRF size; (d\&e) Statistical histogram of receptive classical receptive field size and extra-classical receptive field of all recorded neurons $(\mathrm{N}=40)$.

After calculating the value of $R_{c}$ and $R_{e}$ for each recording site, we mapped their new size-tuning curves in the similar way with bars of one side length fixing at $R_{c}$ ( or $\left.R_{e}\right)$, and the other side length varying from 1 to 4 degree, the same levels as in Fig.1A. The stimuli modes were shown in Fig.2a, in which the solid round indicates the range of CRF and the dotted circle indicates that of eCRF. The size-tuning curves were obtained in the similar way and were both compared with that derived from different sizes of squares. All three curves were normalized to their maximum values respectively. The comparison results were shown in Fig.2b. From Fig.2b, we can see that, the size of stimuli that evoked the maximum response was similar among all three curves, while the suppression degree by larger stimuli was much different with each other. We then calculated suppression index (SI) in different flash stimuli conditions for all selected recording sites $(\mathrm{N}=40)$, and plotted SIs of flashed bars against those of flashed squares. The scatters were shown in Fig.2c, in which red dots indicated results of flashed bars with one side fixing at $R_{c}$ and blue dots indicated those of flashed bars at Re. From the comparison result we can see that, most dots (39 out of 40 for $R_{c}$ and 38 out of 40 for Re, Wilcoxon signed-rank test, $\mathrm{p}<0.01$ ) were below the diagonal line, indicating much stronger surround suppression resulted from flashed squares than from flashed bars. There was no significant difference between surround suppression induced by bars of different widths. The results implied that surround suppression was more intense when surrounding of CRF of tectal neurons was fully stimulated meanwhile than partially stimulated along one side, no matter whether the short side of the bar only covered the CRF of tectal neuron or extend to their eCRF. 


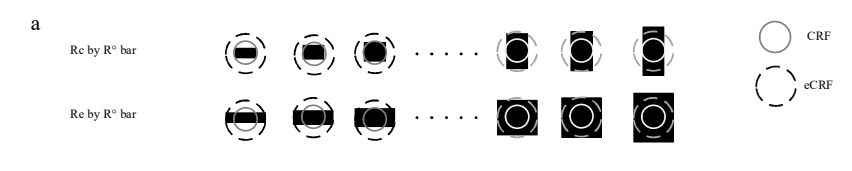

b

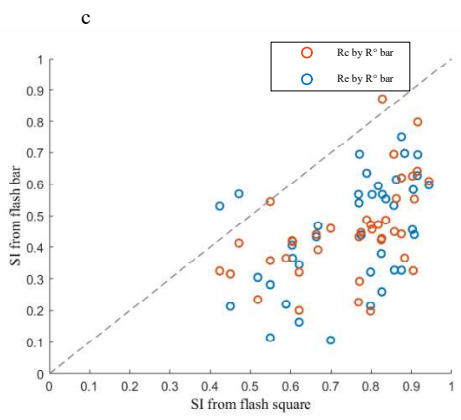

Figure 2. Summarized results of surround suppression induced by flashed bars and the statistical comparison with that by flashed squares. (a) Bars of different length-width ratios by fixing one side at Rc (top row) or Re (bottom row), and varying the length of the other side from 1 to 4 degree. The solid line circle in each subfigure indicates the CRF area and dotted circle indicates the eCRF area, which is the same as in Fig3. a, b \&c. The black square in each subfigure denotes varying lengths of flash bars; (b) The fitted size-tuning curves under each stimulus shown in a (red: adjust-R2 $=0.9049$,left; adjust-R2 $=0.8737$,right; blue: adjust-R2 $=0.9796$,left; adjust-R2 $=0.8354$,right) as well as in Fig1a. (black: adjust-R2=0.9462, left; adjust-R2=0.8628,right); (c) The suppression index for flash square stimulus versus the flash bar stimulus. Each dot represents results from a single recording site. Red symbols show results of neurons response for Rc by $\mathrm{R}$ bar, and blue for Re by $\mathrm{R}$ bar. The diagonal line displays the locus of equal value.

Surround modulation properties by moving stimuli.

Surround modulation properties were further tested with moving squares, with the similar levels of sizes as flashed squares, at 64 recording site, out of which 15 cells were tested in both flashed and moving paradigms. Each stimulus moves at a constant speed $(8 \%$ s, one pixel per frame, refresh rate of $100 \mathrm{~Hz})$ through the RF center from one side to the other side alone a vertical or horizontal direction. In order to make a comparison with results of flashed stimuli, we only considered the cells presenting suppression properties by surrounding moving stimuli. About 46 neurons (71.9\%) were finally selected to make further analysis.

The detail results from an example single-unit recording were summarized in Fig. 3 , in which Fig.3a presented the stimuli modes as well as the raster of their evoking spike train for each repeat and each size level. From Fig.3a we can see that as the size of moving squares grew larger the neuron fired more spikes when the moving square crossed the RF border than through the RF center. Since the path length of each square moving across the RF, which related to the size of the moving stimuli, may affect the response, we calculated the mean firing rate within different widths of time window (bin $1=60 \mathrm{~ms}$, bin $2=160 \mathrm{~ms}$, bin $3=240 \mathrm{~ms}$, bin $4=320 \mathrm{~ms}$, denoted with pairs of dotted lines in different colors in Fig.3a) centered on the moment when the square center passed through the RF center. The size tuning curves were then obtained based on the calculated mean firing rate within different bins. And surround suppression index (SI) was calculated in the similar way. The normalized size tuning curves for different bins were compared, as shown in Fig.3b. The suppression grew weaker as the bin size increased. The surround suppression induced by moving stimuli was the strongest when the stimuli passed near the RF center. 



Figure 3. Summarized results of surround suppression by moving squares. (a) Visual stimuli of different size moving square, and their corresponding firing spike train in 20 repeats. The solid line circle in each subfigure indicates the CRF area and dotted circle indicates the eCRF area. The black square beside each subfigure denotes varying size moving square. The pairs of dotted lines in different colors denoted different widths of time window in which the mean firing rate was calculated; (b) The size-tuning curves under different widths of time window. (Bin1: adjust-R2=0.9837, left; adjust-R2=0.9553, right; Bin2: adjust-R2=0.9796, left; adjust-R2=0.9819, right; Bin3: adjust-R2=0.8733, left; adjust-R2=0.9123, right; Bin4: adjust-R2=0.8925, left; adjust-R2=0.8815).

Note that, the neurons we selected were all unselective to moving direction, thus similar size tuning curves were obtained when the moving direction wsa changed (see Fig.S1 in Supplemental Materials).

\section{Comparison of surround suppression by moving and flashed stimuli}

Finally, we compared the surround suppression properties obtained from flashed and moving stimuli. The calculated SI based on response within different bin sizes in moving paradigm was receptively compared with those of flashed stimuli. Figure $4 a$ presented the boxplot graphs of different groups of data. The comparison results showed that when the bin size was much short, which means the squares just moved near the RF center, the surround suppression derived from moving stimuli has no significantly different from that from flashed stimuli, which also flashed at the cell's RF center. However, when the bin size was a little larger, in which case the response to stimuli moving across the RF border was also taken into account, the quantified suppression degree became weaker than that in flashed condition and moving condition with smaller bin size (Wilcoxon rank sum test, $\mathrm{p}<0.01$ ). When a square moves across the RF boundary of a cell, its RF was just partially stimulated. In such condition, the re- 
sponse was a litter larger, resulting in weaker surround suppression, which was consistent with the phenomenon found in flashed stimuli condition.

Furthermore, the distributions of the CRF and eCRF size obtained from tuning curves in flashed conditions were compared with those from moving stimuli. The comparison results were shown in Fig.4b\&c and indicated that there was no significant difference between results of the two stimuli conditions.

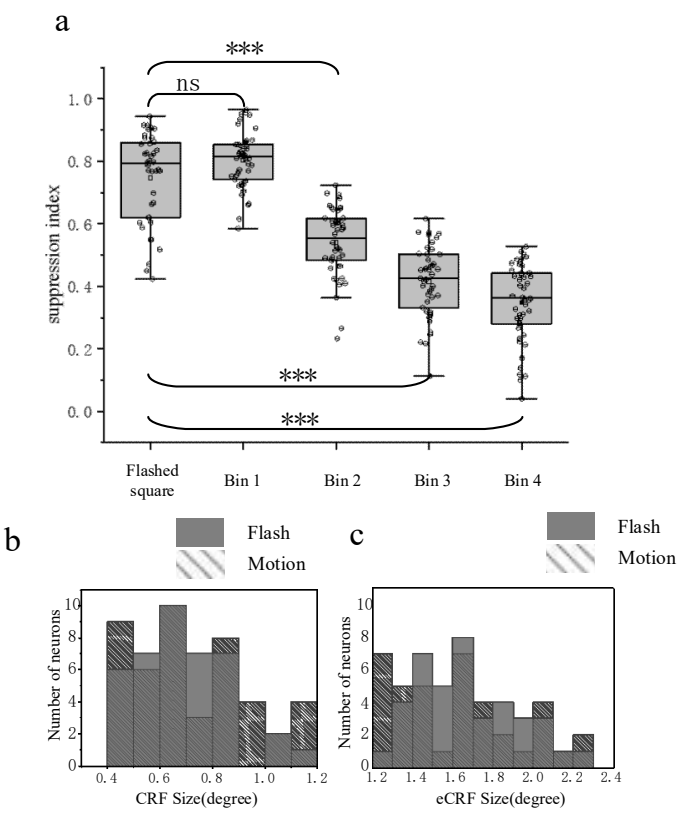

Figure 4. Statistical comparison of surround suppression by flashed and moving stimuli. (a) A. The boxplot graphs for suppression indexes of neural response to moving square stimulus in different widths of time window and to flashed square stimulus. The horizontal line indicates the median of each group of data and the whiskers indicate the lowest and highest point within $1.5 \times$ the interquartile ranges of the lower or upper quartile, respectively. The 'ns' indicates no significantly difference between two groups of data (Wilcoxon rank sum test, $p>0.1$ ), and ${ }^{* * * *}$ indicates significantly difference between two groups of data (Wilcoxon rank sum test, $\mathrm{p}<0.01$ ); (b) Distribution of CRF size for all recorded sites estimated in flashed and moving conditions, respectively. Gray filled rectangle indicates results in flashed conditions, while texture filled rectangle indicates those in moving conditions. (c) Distribution of eCRF size for all recorded sites estimated in flashed and moving conditions, respectively. Gray filled rectangle indicates results in flashed conditions, while texture filled rectangle indicates those in moving conditions.

\section{Discussion}

Current studies on surrounding modulation property of tectal neuron in non-mammals have emphasized their response to contrasting stimuli between center and surrounding under different contrasting conditions, such as different moving directions, different luminance levels and different bar orientation and so on. It could not be inferred from the existing literatures whether the tectal neurons would be inhibited when the surrounding of their CRF was stimulated partially stimulated along one side compared with all around stimulation. In addition, it was not known whether moving objects have the same inhibitory effect as flashing ones. In the present study, we answered these questions. Our results showed that most of tectal neurons in pigeon presented similar surround modulation upon their response to moving and flashed stimuli. Both responses presented suppressive rather than facilitative, which were first enhanced and then suppressed as the size of stimuli increased. The suppression was more intense when the surrounding of CRF of tectal neurons was fully stimulated than partially stimulated. 
The receptive fields in pigeon OT were firstly marked in 1970s [40,41], and the existence of the center-surround structure was tested by using a wider moving or flashing bars than the excitatory area of the field in the subsequent studies [42-44]. Suppressive effects in our study are consistent with those findings suppressive effects. However, they showed standard (center-surround) structure by simple using moving or flashing bars and being short of a quantitative comparison between them. Recent evidence suggests that tectal neurons have an excitatory receptive field surrounded by an inhibitory receptive field and showed regional variation in the dorsal, dorso-lateral, lateral and ventro-lateral tectum [45], which also accorded with our recording location. What's more we found that the surround suppression induced by square extending outside the RF was significantly larger than that by bars with only one side extending outside the RF. The results implied potential spatial arrangement of lateral inhibition from other areas, which still requires further confirmation. Nevertheless, early studies have speculated that the inhibition may be from retina and transmitted to the tectal cells by way of both, feedforward and feedback pathways [46-48]. The subsequent studies showed excitatory and inhibitory RF of tectal cells were differentially modified by magnocellular and parvocellular divisions of the pigeon nucleus isthmi $[49,50]$. Our study supplemented the existing studies on lateral inhibition presented in tectal neurons, and deepened our understanding of information transmission rules in avian midbrain.

Note that a number of earlier studies have reported that most tectal neurons were sensitive to moving stimuli but not responded to stationary ones, and only small number of tectal neurons responded to both moving and flashed stimuli $[32,40,41,51]$. However, in our study we found lots of the latter kind of neurons, and make a further comparison upon surround suppression induced by moving stimuli and by flashed ones. The unicity of the recorded neuronal response may due to our relative fixed recording site, the dorso-lateral tectal section that can be easily accessed by a simple craniotomy [52]. The recording site and the corresponding neuronal response were quite consistent with that reported earlier [45].

The surround modulation properties were explored in another way by other researchers using groups of bars with different types of center-surround contrast, including moving direction, bar orientation and luminance [34-36]. The results showed that tectal neuronal response was suppressed when the visual stimuli of the surrounding were consistent with that of the center. Obviously the tectal neuronal response was pop out when the contrast between center and surround was moving direction and luminance. Most recent work has also compared the suppression induced by luminance and moving direction, which represented the static and motion feature of visual object respectively, and showed that suppression resulting from motion direction was quite smaller than that from luminance[36]. The results were quite consistent with ours, which showed that the suppression induced by flashed stimuli was stronger than that by moving stimuli when the bin width was large.

\section{Conclusions}

We studied thoroughly in surround modulation properties of tectal neurons in pigeons. The result showed that most neurons presented similar surround suppression properties both in flashed and motion paradigms. Both responses were first enhanced and then suppressed as the size of stimuli increased. The property was consistent with those found in mammals V1, LGN and SC with drifting sinusoid gratings as stimuli. Another finding that fully surround suppression was stronger than partially surround suppression was novel and complemented the existing studies. Furthermore, this study provides a deeper insight into possible hypotheses upon the spatial arrangement of lateral inhibitions from feedback or feedforward streams, which would deepen our understanding of visual information transmission way in the tectofugal pathway of the avian. In addition, the stronger fully surround suppression, corresponding to global inhibition [53], can enhance the saliency degree of visual objects and may help animals to detect 
small objects more easily, which is much important for their survivals in complex nature environment.

Supplementary Materials: The following are available online at www.mdpi.com/xxx/s1, Figure S1: An example result of surround suppression by moving squares in another different moving direction.

Author Contributions: Conceptualization, X.N. and S.H.; methodology, S.H.; software, M.Z; validation, X.N., Z.W. and L.S.; formal analysis, M.Z.; investigation, X.N.; resources, L.S.; data curation, S.H.; writing-original draft preparation, S.H.; writing-review and editing, X.N.; visualization, Z.W; supervision, L.S.; project administration, L.S.; funding acquisition, L.S. All authors have read and agreed to the published version of the manuscript.

Ethics approval and consent to participate: All experiments were performed in accordance with the Society's Policies on the Use of Animals and Humans in Neuroscience Research and approved by the Life Science Ethical Review Committee of Zhengzhou University (No SYXK 2019-0002).

Funding: This research was funded by the National Natural Science Foundation of China (grant number: 61673353), the Key Scientific Research Projects of Colleges and Universities in Henan province (grant number: 20A413009), and the Key Discipline Construction Project of Zhengzhou University in 2019 (project number: XKZDQY201905).

Institutional Review Board Statement: All experiments were performed in accordance with the Guidelines of the Care and Use of Laboratory Animals Monitoring Committee of Zhengzhou University, and Experiments were approved by the ethics committee at Zhengzhou University.

Data Availability Statement: The datasets during and analysed during the current study available from the corresponding author on reasonable request.

Acknowledgments: This study was supported by the National Natural Science Foundation of China (grant number: 61673353), the Key Scientific Research Projects of Colleges and Universities in Henan province (grant number: 20A413009), and the Key Discipline Constructon Project of Zhengzhou University in 2019 (project number: XKZDQY201905).

Conflicts of Interest: The authors declare that they have no competing interests.

\section{Appendix A}

Figure S1. An example result of surround suppression by moving squares in another different moving direction.

A. Visual stimuli of different size moving square, as well as their corresponding firing spike train in 20 repeats. The solid line circle in each subfigure indicates the CRF area and dotted circle indicates the eCRF area. The black square beside each subfigure denotes varying size moving square. The pairs of dotted lines in different colors denoted different widths of time window in which the mean firing rate was calculated.

B. The size-tuning curves under different widths of time window. ((Bin1: adjust-R2=0.9867, left; adjust-R2=0.9325, right; Bin2: adjust-R2=0.9778, left; adjust-R2=0.9592, right; Bin3: adjust-R2=0.9817, left; adjust-R2=0.9447, right; Bin4: adjust-R2=0.9827, left; adjust-R2=0.9246). 


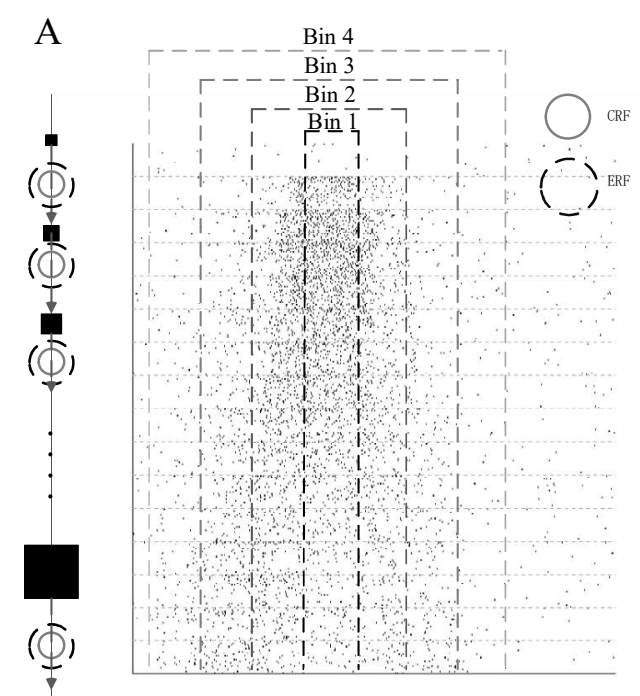

B

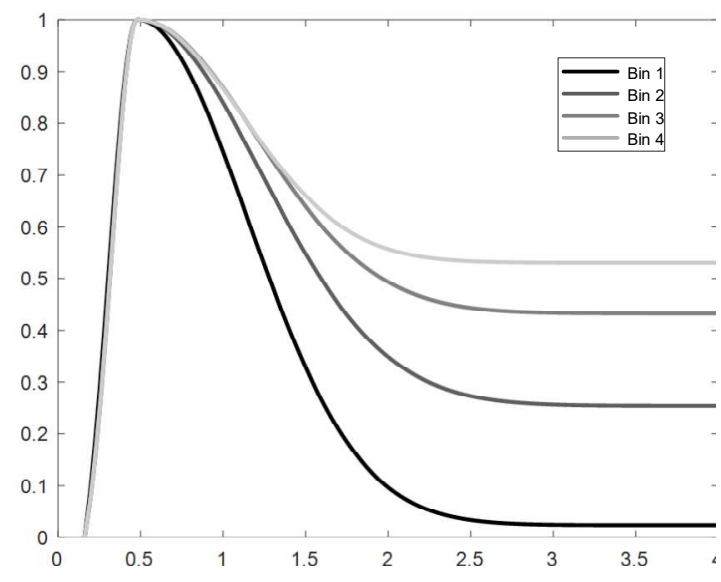

\section{References}

1. Barlow, H. B., (1953), Summation and inhibition in the frog's retina. The Journal of Physiology, 119, 69-88. doi: 10.1113/jphysiol.1953.sp004829.

2. Hubel, D. H., \& Wiesel, T. N. (1965). Receptive fields and functional architecture in two nonstriate visual areas (18 and 19) of the cat. Journal of Neurophysiology, 28(2), 229-289.

3. Cavanaugh JR, Bair W, (2002) Movshon JA. Nature and interaction of signals from the receptive field center and surround in macaque V1 neurons. J Neurophysiol, 88, 2530-2546. doi: 10.1152/jn.00692.2001.

4. Blakemore C., Tobin E. A. (1972). Lateral inhibition between orientation detectors in the cat's visual cortex. Exp. Brain Res. 15, 439-440. doi: 10.1007/BF00234129

5. Nelson JI, Frost BJ (1978) Orientation-selective inhibition from beyond the classic visual receptive field. Brain Res 139:359-365.

6. Knierim JJ, Van Essen DC (1992) Neuronal responses to static texture patterns in area V1 of the alert macaque monkey. J Neurophysiol 67:961-980.

7. DeAngelis GC, Freeman RD, Ohzawa I (1994) Length and width tuning of neurons in the cat's primary visual cortex. J Neurophysiol 71:347-374.

8. Sengpiel F, Sen A, Blakemore C (1997) Characteristics of surround inhibition in cat area 17. Exp Brain Res 116:216-228.

9. Seriès P, Lorenceau J , Frégnac, Y. (2003), The "silent" surround of V1 receptive fields: theory and experiments[J]. Journal of Physiology Paris, 97(4-6):453-474.

10. Sceniak MP, Ringach DL, Hawken MJ, Shapley R (1999) Contrast's effect on spatial summation by macaque V1 neurons. Nat Neurosci 2:733-739.

11. Walker GA, Ohzawa I, Freeman RD (1999) Asymmetric suppression outside the classical receptive field of the visual cortex. J Neurosci 19:10536-10553.

12. Walker GA, Ohzawa I, Freeman RD (2000) Suppression outside the classical cortical receptive field. Vis Neurosci 17:369 -379. 
13. Akasaki T, Sato H, Yoshimura Y, Ozeki H, Shimegi S (2002) Suppressive effects of receptive field surround on neuronal activity in the cat primary visual cortex. Neurosci Res 43:207-220.

14. Maffei L, Fiorentini A (1976) The unresponsive regions of visual cortical receptive fields. Vision Res 16:1131-1139.

15. Li CY, Li W (1994) Extensive integration field beyond the classical receptive field of cat's striate cortical neurons- classification and tuning properties. Vision Res 34:2337-2355.

16. Sillito AM, Grieve KL, Jones HE, Cudeiro J, Davis J (1995) Visual cortical mechanisms detecting focal orientation discontinuities. Nature 378:492-496.

17. Levitt JB, Lund JS (1997) Contrast dependence of contextual effects in primate visual cortex. Nature 387:73-76.

18. Polat U, Mizobe K, Pettet MW, Kasamatsu T, Norcia AM (1998) Collinear stimuli regulate visual responses depending on cell's contrast threshold. Nature 391:580 -584.

19. Albright, T. D., \& Stoner, G. R. (2002). Contextual influences on visual processing. Annual review of neuroscience, 25, 339379.

20. Nomura, T., \& Izawa, E. I. (2017). Avian brains: Insights from development, behaviors and evolution. Development, growth \& differentiation, 59(4), 244-257.

21. Shimizu, T., \& Bowers, A. N. (1999). Visual circuits of the avian telencephalon: evolutionary implications. Behavioural brain research, 98(2), 183-191.

22. Soto, F. A., \& Wasserman, E. A. (2014). Mechanisms of object recognition: what we have learned from pigeons. Frontiers in neural circuits, 8, 1-22.

23. Cavanaugh, J. R., Bair, W., \& Movshon, J. A. (2002). Selectivity and spatial distribution of signals from the receptive field surround in macaque V1 neurons. Journal of neurophysiology, 88(5), 2547-2556.

24. Webb BS, Tinsley CJ, Vincent CJ, Derrington AM (2005) Spatial distribution of suppressive signals outside the classical receptive-field in lateral geniculate nucleus. J Neurophysiol 94: 1789-1797.

25. Barchini, J., Shi, X., Chen, H., \& Cang, J. (2018). Bidirectional encoding of motion contrast in the mouse superior colliculus. eLife, 7, e35261.

26. Okamoto, M., Naito, T., Sadakane, O., Osaki, H., \& Sato, H. (2009). Surround suppression sharpens orientation tuning in the cat primary visual cortex. The European journal of neuroscience, 29(5), 1035-1046.

27. Chen, G., Dan, Y. and Li, C.-Y. (2005), Stimulation of non-classical receptive field enhances orientation selectivity in the cat. The Journal of Physiology, 564: 233-243.

28. Xing, D. , Shapley, R. M. , Hawken, M. J. , \& Ringach, D. L. (2005). Effect of stimulus size on the dynamics of orientation selectivity in macaque v1. Journal of Neurophysiology, 94(1), 799-812.

29. Niu, X., Shi, L., Wan, H., Wang, Z., Shang, Z., \& Li, Z. (2015). Dynamic functional connectivity among neuronal population during modulation of extra-classical receptive field in primary visual cortex. Brain research bulletin, 117, 45-53.

30. De Franceschi, G., \& Solomon, S. G. (2020). Dynamic Contextual Modulation in Superior Colliculus of Awake Mouse. eNeuro, 7(5), ENEURO.0131-20.2020.

31. Frost, B. J., Scilley, P. L., \& Wong, S. C. (1981). Moving background patterns reveal double-opponency of directionally specific pigeon tectal neurons. Experimental brain research, 43(2), 173-185. https://doi.org/10.1007/BF00237761

32. Frost, B.J., Cavanagh, P. \& Morgan, B. (1988). Deep tectal cells in pigeons respond to kinematograms. J. Comp. Physiol. 162, 639-647. https://doi.org/10.1007/BF01342639.

33. Sun H J , Zhao J, Southall T L , Southall and Xu B. (2002). Contextual influences on the directional responses of tectal, cells in pigeons[J]. Visual Neuroscience, 19(02):133-144.

34. Zahar, Y., Wagner, H., \& Gutfreund, Y. (2012). Responses of tectal neurons to contrasting stimuli: an electrophysiological study in the barn owl. PloS one, 7(6), e39559.

35. Zahar, Yael, Lev-Ari, Tidhar, Wagner, \& Hermann, et al. (2018). Behavioral evidence and neural correlates of perceptual grouping by motion in the barn owl. The Journal of Neuroscience: The Official Journal of the Society for Neuroscience, 38(30), 6653-6664.

36. Niu, X. , Huang, S. , Yang, S. , Wang, Z. , \& Shi, L. . (2020). Comparison of pop-out responses to luminance and motion contrasting stimuli of tectal neurons in pigeons. Brain Research, 1747, 147068.

37. Ozeki H, Sadakane O, Akasaki T, Naito T, Shimegi S, Sato H. (2004). Relationship between excitation and inhibition underlying size tuning and contextual response modulation in the cat primary visual cortex. J Neurosci.;24:1428-1438.

38. Sceniak MP, Hawken MJ, Shapley R (2001) Visual spatial characterization of macaque V1 neurons. J Neurophysiol 85:18731887.

39. Osaki, H., Naito, T., Sadakane, O., Okamoto, M. and Sato, H. (2011), Surround suppression by high spatial frequency stimuli in the cat primary visual cortex. European Journal of Neuroscience, 33: 923-932.

40. Jassik-Gerschenfeld, D, Minois, F, \& Condé-Courtine, F. (1970). Receptive field properties of directionally selective units in the pigeon's optic tectum. Brain research, 24(3), 407-421. https://doi.org/10.1016/0006-8993(70)90182-4

41. Jassik-Gerschenfeld, D., \& Guichard, J. (1972). Visual receptive fields of single cells in the pigeon's optic tectum. Brain research, 40(2), 303-317. https://doi.org/10.1016/0006-8993(72)90136-9

42. Hughes, C. P., \& Pearlman, A. L. (1974). Single unit receptive fields and the cellular layers of the pigeon optic tectum. Brain research, 80(3), 365-377. https://doi.org/10.1016/0006-8993(74)91023-3 
43. Hardy, O., \& Jassik-Gerschenfeld, D. (1980). Spatial organization of the excitatory regions in the receptive fields of the avian optic tectum and their spatial frequency selectivity. Brain research, 199(2), 452-458. https://doi.org/10.1016/0006-8993(80)90703-9

44. Leresche, N., Hardy, O., \& Jassik-Gerschenfeld, D. (1984). Suppressive regions in the visual receptive fields of single cells of the pigeon's optic tectum. Experimental brain research, 53(2), 327-334. https://doi.org/10.1007/BF00238162

45. Gu, Y. , Wang, Y. , \& Wang, S. R. . (2000). Regional variation in receptive field properties of tectal neurons in pigeons. Brain Behavior \& Evolution, 55(4), 221-228.

46. Leresche, N., Hardy, O., Audinat, E., \& Jassik-Gerschenfeld, D. (1986). Synaptic organization of inhibitory circuits in the pigeon's optic tectum. Brain research, 365(2), 383-387.

47. Winkowski, D. E., and Knudsen, E. I. (2006). Top-down gain control of the auditory space map by gaze control circuitry in the barn owl. Nature 439, 336-339.

48. Winkowski, D. E., and Knudsen, E. I. (2007). Top-down control of multimodal sensitivity in the barn owl optic tectum. J. Neurosci. 27, 13279-13291.

49. Wang, Y., Xiao, J., \& Wang, S. R. (2000). Excitatory and inhibitory receptive fields of tectal cells are differentially modified by magnocellular and parvocellular divisions of the pigeon nucleus isthmi. Journal of comparative physiology. A, Sensory, neural, and behavioral physiology, 186(6), 505-511.

50. Niu Y, Xiao Q, Liu R, Wu L, Wang S (2006) Response characteristics of the pigeon's pretectal neurons to illusory contours and motion. The Journal of Physiology 577(3):805-813

51. Frost, B.J. (1978). Moving background patterns alter directionally specific responses of pigeon tectal neurons. Brain Res. 151, 599-603

52. Letelier, J. C., Marin, G., Sentis, E., Tenreiro, A., Fredes, F., \& Mpodozis, J. (2004). The mapping of the visual field onto the dorso-lateral tectum of the pigeon (Columba livia) and its relations with retinal specializations. Journal of neuroscience methods, 132(2), 161-168. https://doi.org/10.1016/j.jneumeth.2003.09.007

53. Mysore, S. P., Asadollahi, A., \& Knudsen, E. I. (2010). Global inhibition and stimulus competition in the owl optic tectum. The Journal of neuroscience : the official journal of the Society for Neuroscience, 30(5), 1727-1738. https://doi.org/10.1523/JNEUROSCI.3740-09.2010 